\title{
Living Donor Liver Transplantation with Renoportal Anastomosis for a Patient with Congenital Absence of the Portal Vein
}

\author{
Hajime Uchida, Seisuke Sakamoto, Takanobu Shigeta, Ikumi Hamano, \\ Hiroyuki Kanazawa, Akinari Fukuda, Chiaki Karaki, Atsuko Nakazawa, and Mureo Kasahara
}

\begin{abstract}
Department of Transplant Surgery, National Center for Child Health and Development, 2-10-1 Okura, Setagaya-ku, Tokyo 157-8535, Japan
\end{abstract}

Correspondence should be addressed to Hajime Uchida, uchida-h@ncchd.go.jp

Received 11 June 2012; Accepted 16 September 2012

Academic Editors: E. Ameh, F. Catena, and A. Cho

Copyright (c) 2012 Hajime Uchida et al. This is an open access article distributed under the Creative Commons Attribution License, which permits unrestricted use, distribution, and reproduction in any medium, provided the original work is properly cited.

\begin{abstract}
A congenital absence of the portal vein (CAPV) is a rare disorder that may lead to an intrapulmonary shunt. A 14-year-old male with CAPV underwent living donor liver transplantation with a left lobe graft from his father. The portal vein reconstruction was achieved with a renoportal anastomosis using an interpositional graft from the native collateral vein, because portal venous system directly drains to the left renal vein without constructing the confluence of superior mesenteric vein and splenic vein. The patient is doing well with a normal liver function and mild hypoxemia.
\end{abstract}

\section{Introduction}

A congenital absence of the portal vein (CAPV) is a rare disorder that may lead to an intrapulmonary shunt. The complete portosystemic shunt not perfusing the liver via portal vein, defined as type I, is especially rare, but recently liver transplantation has been recognized as a curative operation for symptomatic CAPV type I patients with uncontrollable hepatic encephalopathy, pulmonary hypertension, and intrapulmonary shunt and become increasingly reported.

\section{Case Report}

A 14-year-old Japanese boy, who had a corrective surgery for tetralogy of Fallot at age 4, presented with hyperammonemia (serum NH3 $100 \mu \mathrm{mol} / \mathrm{L}$ ), coagulopathy (PTINR 2.19), and hypoxemia $\left(\mathrm{PaO}_{2} 47.2 \mathrm{mmHg}\right)$ and was admitted to the hospital. The patient had been diagnosed with hypergalactosemia by neonatal metabolic screening. At the time of assessment on the age of 14, a laboratory evaluation showed serum NH3 $115 \mu \mathrm{mol} / \mathrm{L}$; serum bilirubin $2.22 \mathrm{mg} / \mathrm{dL}$; AST $30 \mathrm{IU} / \mathrm{L}$; albumin $3.0 \mathrm{~g} / \mathrm{dL}$; total bile acid $131.3 \mu \mathrm{mol} / \mathrm{L}$ (normal range, $10 \mu \mathrm{mol} / \mathrm{L}$ ). He presented dyspnea with prominent clubbed fingers and mild lip cyanosis and had been on long-term oxygen therapy at home. Further imaging studies revealed CAPV with a huge splenorenal shunt (Figure 1(a)), retrograde flow of a dilated collateral vein $(7.5 \mathrm{~cm}$ in length) which could be used as a vein graft (Figure 1(b)), and severe intrapulmonary shunting (IPS: shunt ratio $56.0 \%$ by lung perfusion scintigraphy with $\mathrm{Tc}^{99 \mathrm{~m}}$-macroaggregated albumin) without pulmonary hypertension. The portal venous system directly drains to the left renal vein without constructing the confluence of superior mesenteric vein and splenic vein. The shunt vessels were multifocal, and the ligations of these vessels were difficult to indicate.

Though lactulose had been used to treat hyperammonemia, serum ammonia levels were still high. Due to recurrent hyperammonemia and progressive IPS despite medical treatment and protein restriction, the patient underwent living donor liver transplantation (LDLT).

The donor was the patient's 39-year-old father with an incompatible blood type, and the recipient received rituximab for 4 weeks prior to LDLT and preoperative plasma 


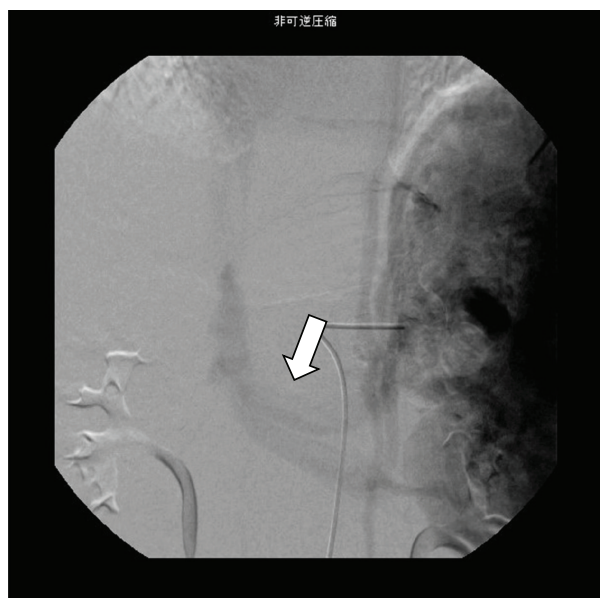

(a) Huge splenorenal shunt

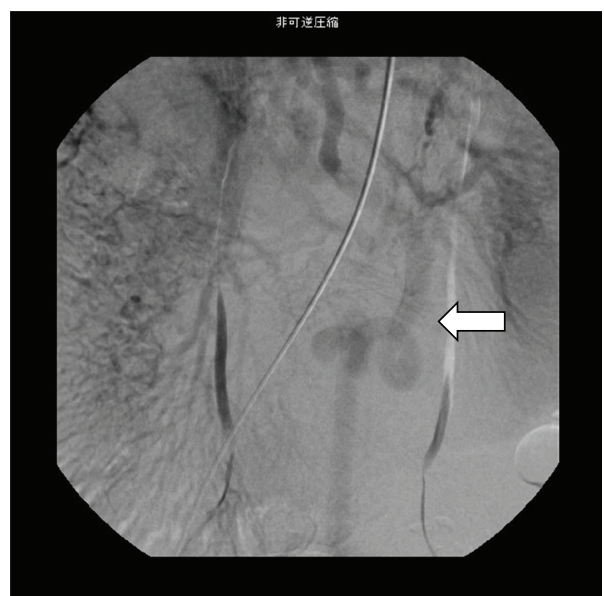

(b) Retrograde flow of inferior mesenteric vein

Figure 1: Preoperative angiography. (a) A huge splenorenal shunt (arrow) drained into the inferior vena cava without entering the hepatic hilum. (b) Retrograde flow in the dilated collateral vein (arrow) was observed.

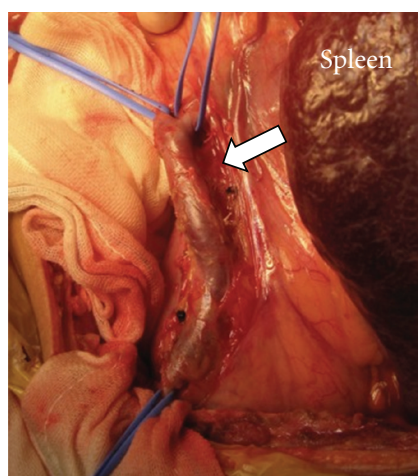

(a) Dilated inferior mesenteric vein

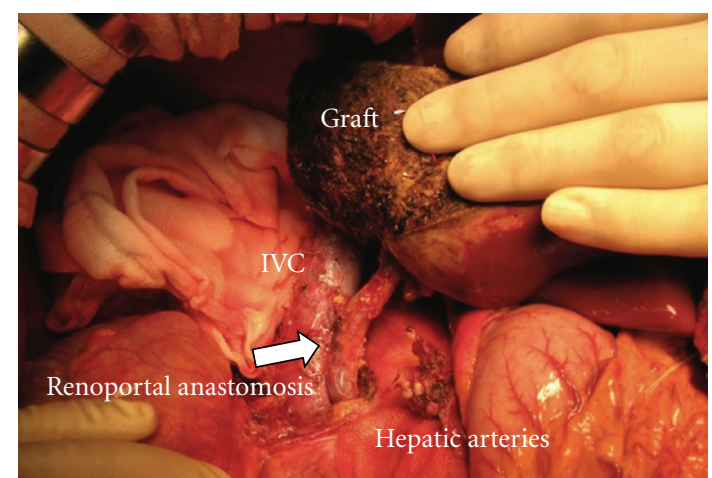

(b) Renoportal anastomosis with native inferior mesenteric vein graft interposition

FIGURE 2: Renoportal anastomosis with the interpositional vein graft. (a) The intraoperative view shows the dilated collateral vein (arrow) procured as an interposition vein graft. (b) After the completion of the renoportal anastomosis with the vein graft (arrow).

exchange to reduce the complications related to $\mathrm{ABO}$ incompatibility [1]. The anti-A IgM/IgG titer was successfully decreased from $64 / 32$ to $2 / 2$ at the time of LDLT. A liver graft left sector weighing $485 \mathrm{~g}$, representing $1.41 \%$ of the graft-to-recipient weight ratio, was procured. The recipient laparotomy showed CAPV, a splenorenal shunt, and retrograde flow of the collateral vein (Figure 2(a)). The recipient hepatectomy was uncomplicated. A histological examination of the $618 \mathrm{~g}$ explanted symmetrical native liver, which was $70.2 \%$ of the estimated standard liver volume, showed atrophic portal veins visible in the portal tracts.

The superior mesenteric vein and splenic vein directly drained into the left renal vein without entering the hepatic hilum. After the procurement of a $7.5 \mathrm{~cm}$ collateral vein for an interposition vein graft for the portal anastomosis without any noted circulatory changes, the left renal vein was divided with an autovascular stapler (PROXIMATE TX Reloadable Linear Staplers; Ethicon, USA) at its junction with the inferior vena cava (IVC). The trunk of the left renal vein, $20 \mathrm{~mm}$ in diameter, was anastomosed with the interposition graft with 6-0 PDS interrupted sutures. The vein graft was turned upward beside the duodenum and anastomosed directly to the graft portal vein in an end-to-end fashion with sufficient forward flow (flow volume, $921 \mathrm{~mL} / \mathrm{min}$ ) (Figure 2(b)). Mesenteric venous congestion did not develop. The operation lasted for 10 hours and 36 minutes, and blood loss was 1,669 mL. At the 3-year followup, the patient was doing well with a normal liver/kidney function with mild hypoxemia (shunt ratio of $35.0 \%$ ).

\section{Discussion}

CAPV is a rare disorder that may lead to hepatic encephalopathy and IPS. The portal vein derives from, embryologically, selective involution and the vitelline venous system, and its abnormality may result in CAPV. 
Some patients with CAPV were diagnosed at the time of neonatal screening for hypergalactosemia. LT is indicated as a curative operation for CAPV for uncontrollable hepatic encephalopathy, pulmonary hypertension, and IPS. Although pulmonary hypertension was not seen in the present patient, pathophysiology of pulmonary hypertension in CAPV is demonstrated as thromboembolic pulmonary arterial hypertension, and this state could be cured if the shunt vessel is able to close. Recently, preemptive LT for CAPV patient has become increasingly reported, because LT is the only therapeutic option to prevent regression of progressive pulmonary hypertension and IPS [2].

Securing adequate portal venous flow is crucial for successful LT. However, anastomosis between the graft portal vein and native vein may not be possible in patients with extensive portal thrombosis and/or in the absence of a proper tributary of the portal venous system $[3,4]$. In the present patient, having CAPV with a significant splenorenal shunt and without confluence of superior mesenteric vein and splenic vein, the renoportal anastomosis was indicated. Renoportal anastomosis for liver allografts in cases with thrombosed PV and splenorenal/mesenteric-renal shunting has been described with excellent long-term outcome [510]. To the best of our knowledge, this is the first report of renoportal anastomosis in LDLT for CAPV. The renoportal anastomosis for the patient with CAPV with a significant splenorenal shunt appears to be a safe and feasible technique, which should be considered as a treatment option in LDLT.

\section{Abbreviations}

CAPV: Congenital absence of the portal vein

HPS: Hepatopulmonary syndrome

IPS: Intrapulmonary shunting

IVC: Inferior vena cava

LDLT: Livingdonor liver transplantation

LT: Liver transplantation

PT-INR: International normalized ratio of prothrombin time

$\mathrm{PaO}_{2}$ : $\quad$ Partial pressure of arterial oxygen

AST: $\quad$ Aspartate aminotransferase.

\section{Acknowledgments}

This work was supported in part by grants from the Scientific Research Fund of the Ministry of Education and by a Research Grant for Immunology, Allergy and Organ Transplant from the Ministry of Health, Labor and Welfare, Japan (nos. 21591403, 21-04, and 21-042) and the Foundation for Growth Science, Japan.

\section{References}

[1] H. Egawa, S. Teramukai, H. Haga, M. Tanabe, M. Fukushima, and M. Shimazu, "Present status of ABO-incompatible living donor liver transplantation in Japan," Hepatology, vol. 47, no. 1, pp. 143-152, 2008.

[2] T. Ohno, J. Muneuchi, K. Ihara et al., "Pulmonary hypertension in patients with congenital portosystemic venous shunt: a previously unrecognized association," Pediatrics, vol. 121, no. 4, pp. e892-e899, 2008.

[3] T. Kato, D. M. Levi, W. Defaria, S. Nishida, and A. G. Tzakis, "Liver transplantation with renoportal anastomosis after distal splenorenal shunt," Archives of Surgery, vol. 135, no. 12, pp. 1401-1404, 2000.

[4] A. G. R. Sheil, M. S. Stephen, A. K. K. Chui, J. Ling, and M. J. Bookallil, "A liver transplantation technique in a patient with a thrombosed portal vein and a functioning renal-lieno shunt," Clinical Transplantation, vol. 11, no. 1, pp. 71-73, 1997.

[5] C. O. Esquivel, G. Klintmalm, S. Iwatsuki et al., "Liver transplantation in patients with patent splenorenal shunts," Surgery, vol. 101, no. 4, pp. 430-432, 1987.

[6] G. Manzanet, F. Sanjuán, P. Orbis et al., "Liver transplantation in patients with portal vein thrombosis," Liver Transplantation, vol. 7, no. 2, pp. 125-131, 2001.

[7] D. Azoulay, R. Adam, D. Castaing et al., "Liver transplantation with cavoportal or renoportal anastomosis: a solution in cases of diffuse portal thrombosis," Gastroenterologie Clinique et Biologique, vol. 26, no. 4, pp. 325-330, 2002.

[8] M. Paskonis, J. Jurgaitis, A. Mehrabi et al., "Surgical strategies for liver transplantation in the case of portal vein thrombosis-current role of cavoportal hemitransposition and renoportal anastomosis," Clinical Transplantation, vol. 20, no. 5, pp. 551-562, 2006.

[9] A. Miyamoto, T. Kato, K. Dono et al., "Living-related liver transplantation with renoportal anastomosis for a patient with large spontaneous splenorenal collateral," Transplantation, vol. 75, no. 9, pp. 1596-1598, 2003.

[10] S. Marubashi, K. Dono, H. Nagano et al., "Living-donor liver transplantation with renoportal anastomosis for patients with large spontaneous splenorenal shunts," Transplantation, vol. 80, no. 12, pp. 1671-1675, 2005. 


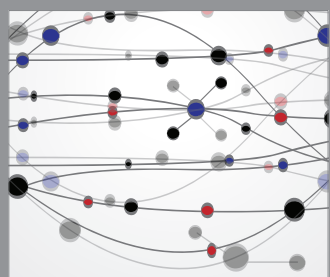

The Scientific World Journal
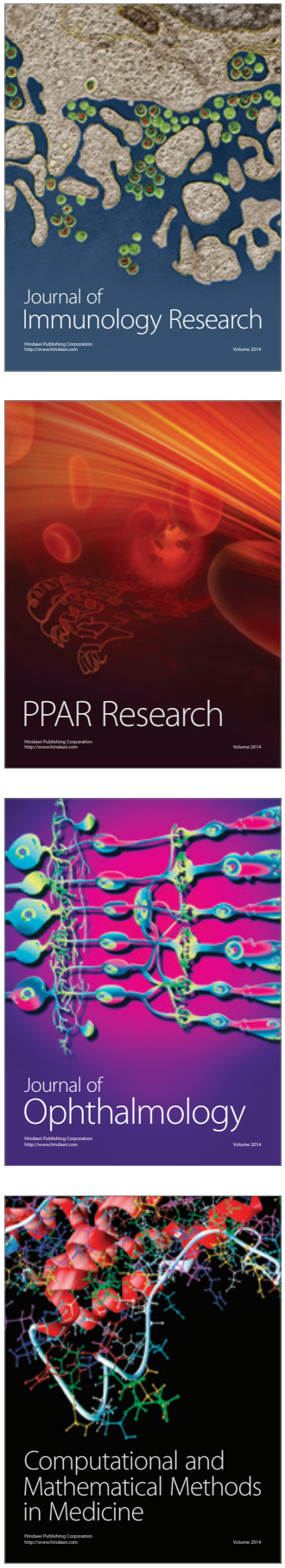

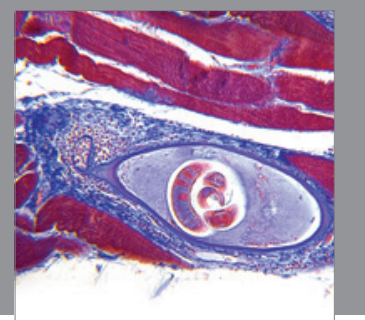

Gastroenterology

Research and Practice
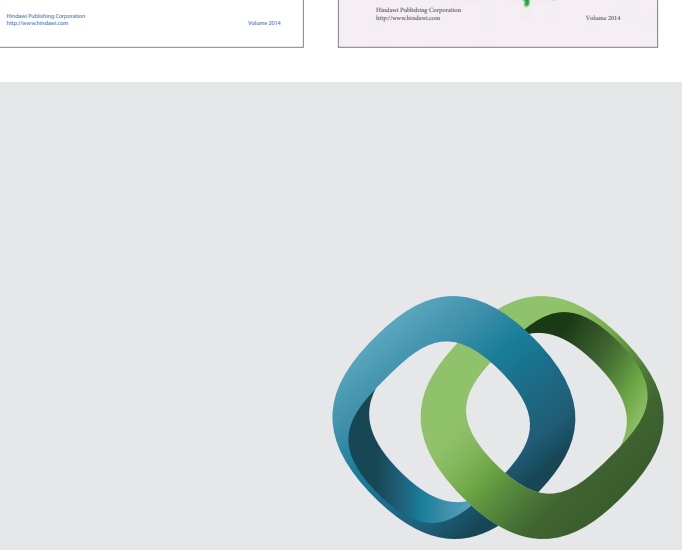

\section{Hindawi}

Submit your manuscripts at

http://www.hindawi.com
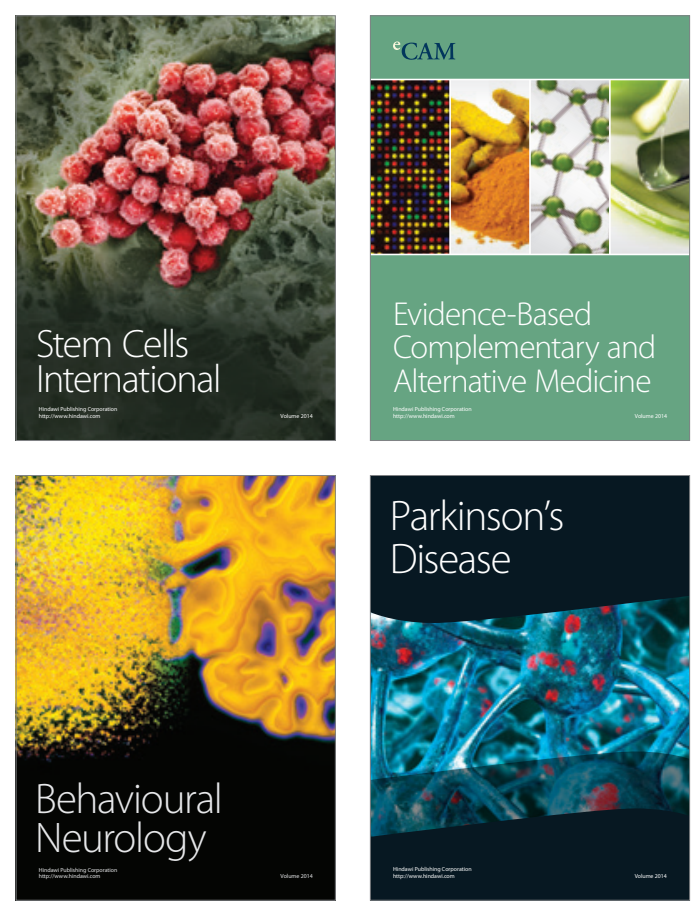

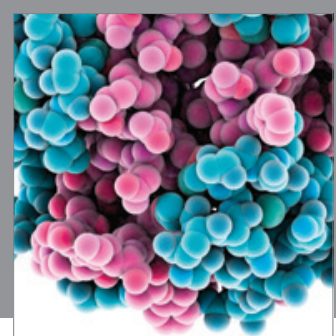

Journal of
Diabetes Research

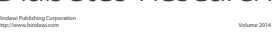

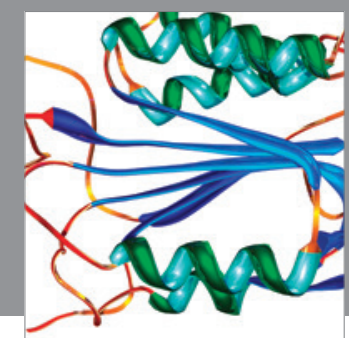

Disease Markers
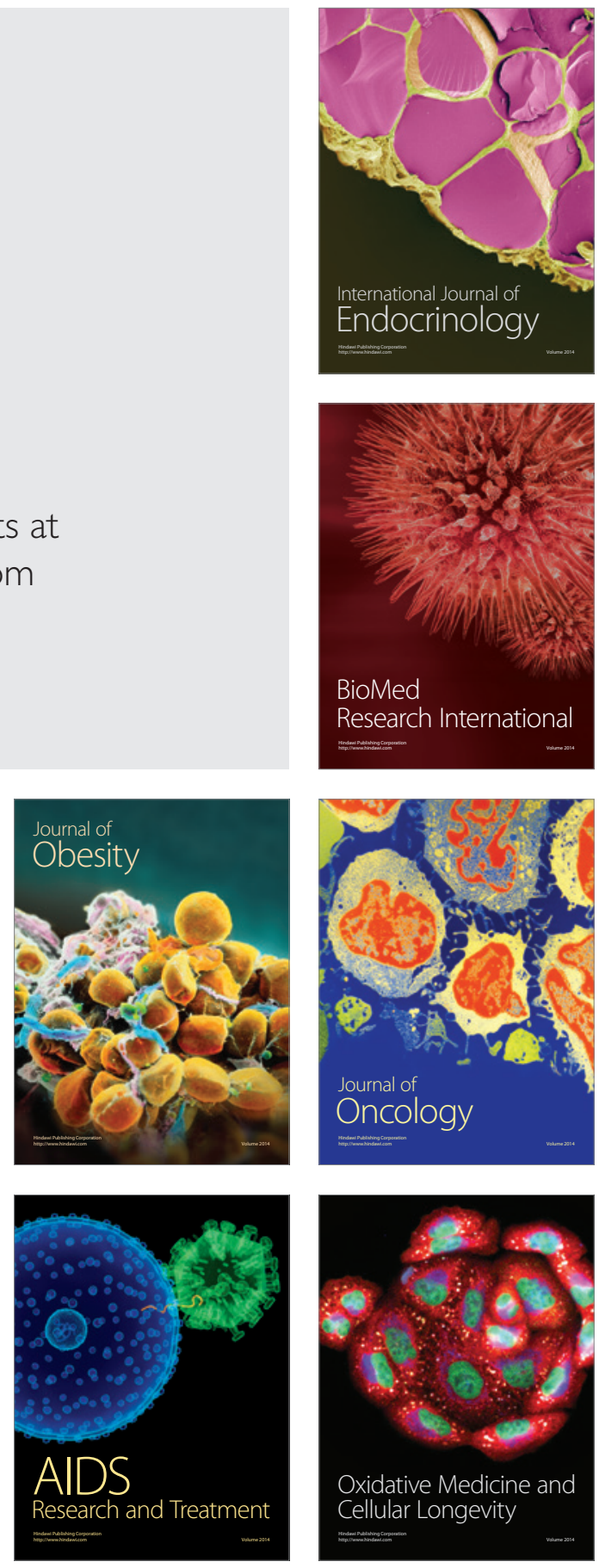